\section{The effect of scopolamine on the Kamin effect: A test of the parasympathetic overreaction hypothesis*}

\author{
RICHARD W. THOMPSON \\ Western Washington State College, Bellingham, Wash. 98225 \\ and \\ CATHY NIELSEN \\ University of Wisconsin-Milwaukee, Milwaukee, Wis. 53201
}

The Kamin effect, the U-shaped curve of retention of a partially learned shuttle avoidance response, was replicated in animals trained and tested for retention after delays of $0,1,4$, or $24 \mathrm{~b}$. Other animals treated with scopolamine hydrobromide, a cholinergic blocker, during the 1-h delay between learning and relearning showed no pecformance decrement. However, animals treated with inethy scopolamine, which does not cross the blood-brain barrier, during the 1-h delay did show the typical Kamin offect decrement in performance. The results were interpreted as supporting a central cholinergic basis for the Kamin effect.

The Kamin effect, the U-shaped curve of retention of an incompletely learned ayoidance response, was first, described by Kamin in 1957 and since then has been replicated in a number of laboratories using a wide variety of procedures (Denny \& Ditchman, 1962; Denny \& 'Thomas, 1960, Brush, Myer, \& Palmer, 1963; Kamin, 1963; Kumar, 1970). The typical Kamin effect experiment involves giving several groups incomplete training of an avoidance response in a shuttlebox and testing for retention by retraining in different groups after delays of $0,1 \cdot 6$, or $24 \mathrm{~h}$. Animals retrained during the 1.6-h periods show poorer performance than those retrained immediately or $24 \mathrm{~h}$ later. Kamin's original explanation for these results was that the poor retention of the 1-6-h groups was a result of forgetting and that the improvement for the longer delay groups was a result of incubation of anxiety or consolidation of the avoidance response. Grossman (1967) has suggested that the Kamin effect may be a result of the transfer of the engram from one storage system to another, the engram being unavailable during the transfer period. Zerbolio (1969) has suggested a similar interpretation.

An alternative interpretation was offered by Denny (1958) and by Denny \& Ditchman (1962), suggesting that the decrement in performance in the 1-6-h period after original learning was a result of the incubation of

*This research was supported by a grant from the Bureau of Faculty Research. Western Washington State College. Portions of this paper were presented at the Midwestern Psychological Association meeting in Cincinnati, Ohio, 1970. The authors wish to thank Henry Waibel and John Dempster for their help in collecting data and Mr. Marvin Guthaus and the Upjohn Co., Kalamazoo, Mich., for supplying the methylscopolamine. anxiety or fear, the animal being incapable of making the adaptive avoidance response during retention testing because of interfering freezing responses. Animals tested immediately after original learning had not had time for the incubation to occur, and animals tested $24 \mathrm{~h}$ after original learning had had the fear incubate and then decline.

A third hypothesis to account for the retention decrement was suggested by Brush, Myer, \& Palmer in 1963. They hypothesized that there was a parasympathetic overreaction to the shock and stress of initial training which reached a maximum at $1-4 \mathrm{~h}$ after original learning, and that, in relearning, this parasympathetic overreaction left the animal "ill equipped to cope with the stress of such training [Brush, Myer, \& Palmer, 1963 , p. 544]."

The present experiment was primarily concerned with testing the parasympathetic overreaction hypothesis by treating animals with the cholinergic blocking drug, scopolamine hydrobromide, prior to retention testing. The scopolamine would block the parasympathetic response and, according to the SUBJECTS

The experiment was conducted in several steps and involved 115 male obtained from Sprague-Dawley, Madison, Wis. The animals were maintained in groups of 5 on ad lib food and water.

\section{APPARATUS}

The apparatus was a standard shuttlebox, $91.4 \mathrm{~cm}$ long, $35.5 \mathrm{~cm}$ high, and $11.4 \mathrm{~cm}$ wide. The apparatus was painted flat black, except for a the length of the box. The floor was of $0.3-\mathrm{cm}$ brass rods, $1.7 \mathrm{~cm}$ center to hypothesis, prevent the Kamin effect. albino rats, $90-120$ days of age, 4-cm-wide Plexiglas window which ran center. Each half of the grid floor could be charged with $1.6-\mathrm{mA}$ dc current from a C. J. Applegate stimulator (Model 228) through a Grason-Stadler grid scrambler. A $65-\mathrm{dB}$ buzzer was mounted at each end of the shuttlebox, and background noise was $55 \mathrm{~dB}$.

\section{PROCEDURE}

All animals were trained to a criterion of three total avoidance responses and, after the appropriate delay interval, were given 30 retraining trials. All animals were given $5 \mathrm{~min}$ of habituation to the apparatus prior to training and 60 sec prior to retraining. A training trial consisted of the onset of the buzzer at the end of the apparatus closest to the rat, followed 5 sec later by the onset of shock. Both buzzer and shock remained on until the animal crossed the midline of the box and were response terminated. The rat could avoid the shock by responding during the 5 -sec CS-US interval. The intertrial interval was 60 sec. Animals treated with scopolamine hydrobromide or methylscopolamine were injected with $0.5 \mathrm{mg} / \mathrm{kg} \mathrm{IP}$, and saline-treated animals were given identical volumes of physiological saline.

The first step was to replicate the Kamin effect. Four groups of eight rats were trained and retrained after delays of 0 (actually $5 \mathrm{~min}$ ), 1,4 , or $24 \mathrm{~h}$. The animals were returned to their home cages during the delay interval. None of these animals were treated chemically, and they were designated control animals. Later, additional animals were run in the 0 -, $1-$, and 24-h groups, so that there were 12 animals in each group. The saline, scopolamine, and methylscopolamine animals were injected $1 \mathrm{~h}$ prior to retraining, i.e., the 1 -h delay animals were injected immediately after training and the 24-h delay animals were injected $23 \mathrm{~h}$ after training. The $\mathbf{N}$ for each group is presented in the results section.

The mean number of trials to criterion of three total avoidance responses for each group, and its respective $\mathrm{N}$, was: Control $0, \mathrm{~N}=12$, $\overline{\mathrm{X}}=18.5 ;$ Control $1, \mathrm{~N}=12, \overline{\mathrm{X}}=17.1$; Control $4, \quad N=8, \quad \bar{X}=16.8$; Control $24, \mathrm{~N}=12, \overline{\mathrm{X}}=17.2$; Saline 1 , $\mathrm{N}=10, \overline{\mathrm{X}}=16.1$; Saline $24, \mathrm{~N}=10$, $\overline{\mathrm{X}}=16.4 ;$ Methylscopolamine $1, \mathrm{~N}=8$, $\bar{X}=22.4 ; \quad$ Methylscopolamine 24, $\mathrm{N}=9, \quad \overline{\mathrm{X}}=19.8 ; \quad$ Scopolamine 1, $\mathrm{N}=16, \quad \overline{\mathrm{X}}=16.8 ; \quad$ Scopolamine 24 , $\mathrm{N}=8, \quad \overline{\mathrm{X}}=17.1 ;$ artd $24-3$ (to be discussed below), $N=10, \bar{X}=20.0$. Analysis of variance of these data revealed no significant differences among the groups $(\mathrm{F}<1, \mathrm{df}=$ $10 / 104$ ), indicating they were well matched on original learning. 


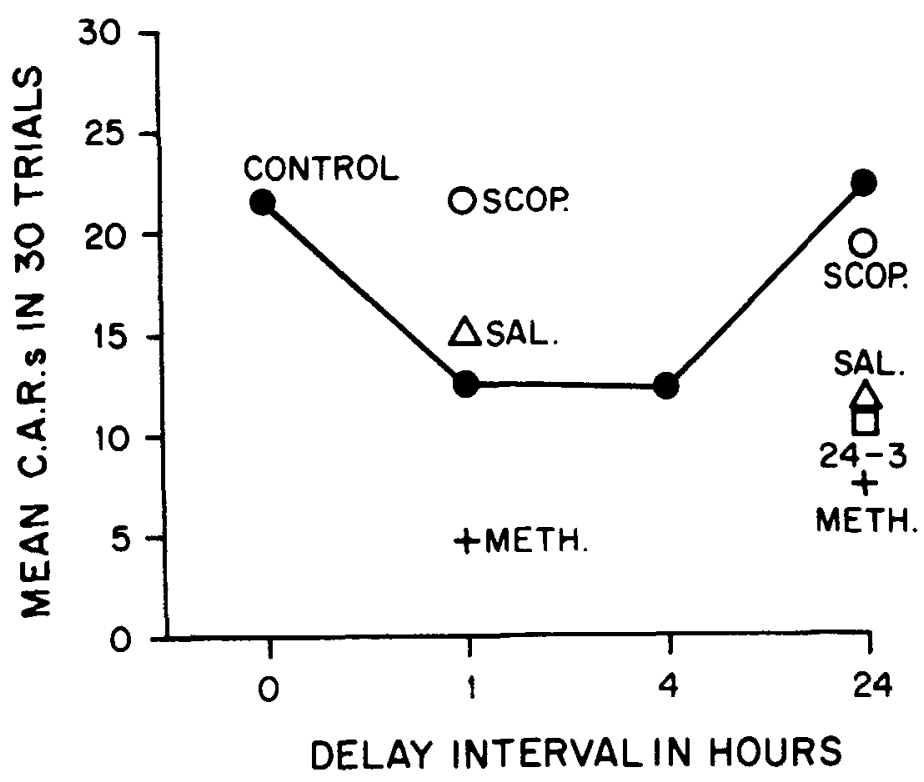

Fig. 1. Mean of the total number of avoidance responses for each group at each interval in relearning.

The mean number of avoidance responses in the 30 relearning trials for each group are presented in Fig. 1. The curve displayed is typical of that found demonstrating the Kamin effect. Analysis of variance of the control animals only on total number of avoidances in the 30 relearning trials indicated that there were significant differences among the various delay intervals $(F=5.49$, $\mathrm{df}=3 / 40, p<.01)$. Subsequent $t$ tests among the various groups indicated no difference between the 0 - and $24 \cdot h$ delay groups or between the 1 - and 4 -h delay groups, but the 1- and 4-h delay groups made significantly fewer avoidance responses than did the 0 - or 24-h delay groups (all ts $\geqslant 2.91$, $\mathrm{df}=40, \mathrm{p}<.01$ ).

Comparisons among the various groups at the 1-h delay interval indicated that the scopolamine-treated animals were superior to both the saline-treated and control animals, which in turn were superior to the methylscopolamine-treated animals ( $F=16.05, \quad \mathrm{df}=3 / 42, \quad \mathrm{p}<.01 ;$ all ts $\geqslant 2.10, \mathrm{df}=42, \mathrm{p}<.05)$.

Similar comparisons among the four groups at the 24-h delay interval indicated no differences between the control and scopolamine-treated animals, nor were there significant differences between the saline- and methylscopolamine-treated animals. However, the control and scopolamine-treated animals made significantly more avoidance responses in relearning than either the saline- or methylscopolamine-treated animals $(\mathrm{F}=16.10, \mathrm{df}=3 / 35, \mathrm{p}<. .01 ; \quad$ all ts $\geqslant 2.86, \mathrm{df}=35, \mathrm{p}<.01)$.

\section{DISCUSSION}

Consideration of the control animals only indicated that the typical $\mathrm{U}$-shaped curve of retention of a partially learned avoidance response, the Kamin effect, was obtained. Saline-treated animals showed the typical poor relearning performance after the 1 -h delay but were also poorer in performance at the 24-h interval than control animals. The latter, unexpected finding will be discussed more fully below. The animals treated with scopolamine at both the 1 - and 24-h retention intervals showed similar performance and, at the 1-h interval, showed significantly better performance than either the control or saline-treated animals. In contrast, the animals treated with methylscopolamine, which does not cross the blood-brain barrier but is only peripherally active, showed significantly poorer performance at the 1-h delay interval than other groups and performance saline-injected animals at the 24-h retention interval.

The results for the scopolamine-treated animals clearly support the hypothesis that the Kamin effect is a result of parasympathetic or cholinergic activity. Manto (1967) has shown that rats injected with saline and epinephrine, or methylscopolamine and epinephrine, show a decrement in activity compared to animals injected with saline, or scopolamine and epinephrine. Manto has argued that high levels of epinephrine cause a parasympathetic overreaction which accounts for the which did not differ from that of lower activity measures. Carlton (1969) has suggested that the same mechanism could account for the Kamin effect, which the present experiment supports. In a sense, then, both the hypothesis of Denny (1958), that the Kamin effect is a result of the incubation of anxiety, and the parasympathetic overreaction hypothesis of Brush, Myer, \& Palmer (1963) are supported. The stress and fear of original learning which is undoubtedly accompanied by strong epinephrine response produces the parasympathetic overreaction which results in freezing behavior (decreased activity) and the decrement in performance during relearning.

The poor performance of the $24-\mathrm{h}$ saline-treated animals led to the speculation that the handling and injection $1 \mathrm{~h}$ prior to relearning may have produced a strong epinephrine response and performance decrement analogous to the Kamin effect. To test this hypothesis, a group of 10 animals were given original learning to a criterion of three total avoidance responses and, $23 \mathrm{~h}$ later, were given three trials on which the CS came on $0.5 \mathrm{sec}$ prior to shock onset. Both buzzer and shock stayed on until the animals escaped, and the two stimuli were response terminated. One hour later, these animals were given 30 standard relearning trials. The results for this group are presented in Fig. 1 and are labeled 24-3. Statistical analysis indicated that this group did not differ significantly from the salineor methylscopolamine-treated animals, but made significantly fewer total avoidance responses than the control or scopolamine-treated animals $(\mathrm{F}=10.24, \mathrm{df}=4 / 44, \mathrm{p}<.001 ;$ both ts $\geqslant 3.19, \quad \mathrm{df}=44, \quad \mathrm{p}<.01)$. The results for the 24-3 group indicate that the poor performance of the salineand methylscopolamine-treated groups may be attributable to a reinstatement of the Kamin effect.

The differences in performance for the scopola mine - and methylscopolamine-treated animals indicates that the Kamin effect is a result of cholinergic activity in the central nervous system, producing inactivity or freezing responses rather than a peripheral parasympathetic effect (Carlton, 1969). The results for methylscopolamine-treated animals indicate that peripheral blocking of the parasympathetic system alone results in impaired shuttlebox performance at both the 1- and 24-h delays. The performance decrement of the 24-h period is confounded with the effects of injections seen in the saline-treated animals, however.

Recent experiments by Hamilton, McCleary, \& Grossman (1968) and by Hamilton \& Grossman (1969) indicate 
that systemic injections or microinjections in the septal area of atropine or scopolamine hydrobromide, both cholinergic inhibitors, result in changes in behavior which are similar to those produced by lesions in the septal area. Animals so treated show poor passive avoidance learning but improved shuttlebox avoidance learning, whereas one-way avoidance learning is unaffected. These results suggest that the septal area may be involved in the Kamin effect. A logical next step would be direct septal microinjections of scopolamine during the 1-6-h retention interval of the Kamin effect design.

The present experiment also suggests that if the strong sympathetic response that accompanies avoidance training could be prevented, then the Kamin effect could also be prevented.

\section{REFERENCES}

BRUSH, R. F., MYER, J. S., \& PALMER, R. F. Effects of kind of prior training and intercession interval upon subsequent avoidance learning. Journal of Comparative \& Physiological Psychology. $1963,56,539-545$.

CARLTON, P. L. Brain-acetylcholine and inhibition. In J. T. Tapp (Ed.), Reinforcement and behavior. New York: Academic Press, 1969. Pp. 286-327.

DENNY, M. R. The "Kamin effect" in avoidance conditioning. American Psychologist, 1958, 13, 419. (Abstract)

DENNY, M, R., \& DITCHMAN, R. E. The focus of maximal "Kamin effect" in rats Journal of Comparative \& Physiological Psychology, 1962, 55, 1069-1070.

DENNY, M. R., \& THOMAS, J. O. Avoidance learning and relearning as a function of shuttlebox dimensions. Science, 1960, 132,620-621.

GROSSMAN, S. P. A textbook of physiological psychology. New York: Wiley, 1967.

HAMILTON, L. W., McClEARY, R. A., \& GROSSMAN, S. P. Behavioral effects of cholinergic septal blockage in the cat. Journal of Comparative \& Physiological Psychology, 1968, 66, 563-568.

HAMILTON, L. W., \& GROSSMAN, S. P. Behavioral changes following disruption of central cholinergic pathways. Journal of Comparative \& Physiological Psychology, 1969, 69, 76-82.

KAMIN, L. J, The retention of an incompletely learned avoidance response. Journal of Comparative \& Physiological Psychology, 1957, 50, 45 7-468.

KAMIN, L. J. Retention of an incompletely learned avoidance response: Some further analysis. Journal of Comparative \& Physiological Psychology, 1963, 56, 713-718.

KUMAR, $R$. Incubation of fear: Experiments on the "Kamin effect" in rats. Journal of Comparative \& Physiological Psychology, 1970, 70, 258-263.

MANTO. P. G. Blockade of epinephrine-induced decrement in activity by scopolamine. Psychonomic Science, 1967, 7, 203-204.

ZERBOLIO, D. J. Memory storage: The first post-trial hour. Psychonomic Science, 1969, 15, 57-58. 